\title{
Preservation of the celiac branch of the vagal nerve for pylorus-preserving gastrectomy: is it meaningful?
}

\author{
Haruna Furukawa $^{1} \cdot$ Manabu Ohashi $^{1} \cdot$ Michitaka Honda $^{1} \cdot$ Koshi Kumagai $^{1} \cdot$ \\ Souya Nunobe ${ }^{1} \cdot$ Takeshi Sano $^{1} \cdot$ Naoki Hiki $^{1}$
}

Received: 12 June 2017 / Accepted: 23 October 2017 / Published online: 10 November 2017

(C) The International Gastric Cancer Association and The Japanese Gastric Cancer Association 2017

\begin{abstract}
Background Preserving the hepatic and pyloric branches of the vagal nerve in laparoscopic pylorus-preserving gastrectomy (LPPG) is considered necessary to maintain the function of the pyloric cuff. However, the clinical benefits of preservation of the celiac branch of the vagal nerve (CBVN) remain unclear.

Methods Of 391 patients who underwent LPPG for early gastric cancer, 116 patients in whom the CBVN was preserved (CBP group) and 58 patients in whom it was not preserved (non-CBP group) were selected through the propensity score-matching method. To evaluate the surgical and oncological safety of preserving the CBVN, postoperative morbidity and mortality were analyzed between these matched groups. Postoperative nutritional status, body weight changes, endoscopic findings, and the incidence of gallstones were compared to evaluate any functional advantages.

Results The short-term surgical outcomes in the CBP group were similar to those in the non-CBP group. The number of dissected lymph nodes did not differ (34 vs. 33.5, $P=0.457)$, and the 5-year recurrence-free survival rates were also similar between both groups $(99.1 \%$ vs. $97.1 \%$, $P=0.844)$. There were no significant differences in postoperative nutritional status, body weight changes, or the incidence of gallstones. By endoscopy, 1 year after surgery
\end{abstract}

Haruna Furukawa and Manabu Ohashi contributed equally to this work.

Naoki Hiki

naoki.hiki@jfcr.or.jp

1 Department of Gastroenterological Surgery, Cancer Institute Hospital, Japanese Foundation for Cancer Research, 3-8-31, Ariake, Kotoku, Tokyo 135-8551, Japan residual food was frequently observed in both groups; however, there were no significant differences in the frequency of remnant gastritis and esophageal and bile reflux.

Conclusions Preserving CBVN in LPPG for early gastric cancer is a feasible procedure. However, no clinical benefits of the preservation of the CBVN after LPPG are identified.

Keywords Early gastric cancer - Laparoscopic pyloruspreserving gastrectomy $\cdot$ Celiac branch of the vagal nerve

\section{Background}

Gastric cancer is one of the most common cancers in the world, with approximately 989,600 new patients and 738,000 deaths per year [1]. In recent years, the number of early gastric cancer (EGC) patients has been increasing, consequent to the development of higher-quality endoscopic instruments, techniques, and mass screening programs initiated by the government [2]. As EGC has a low metastatic incidence and good survival rates after surgery [3-5], the current focus is on less-invasive and function-preserving surgery to improve the postoperative quality of life (QOL).

Pylorus-preserving gastrectomy (PPG) has been widely accepted as one type of function-preserving gastrectomy for EGC located in the middle third of the stomach, and the procedure is now performed through a laparoscopic approach (LPPG) in many institutions [6-8]. Compared with conventional distal gastrectomy, PPG has been reported to have several advantages in postoperative dumping syndrome, including postoperative nutritional status, bile reflux, and prognosis $[9,10]$.

Gastrectomy with preservation of the celiac branch of the vagal nerve (CBVN) for ECG has been also performed as one type of function-preserving gastrectomy in both open 
and laparoscopic techniques $[11,12]$. The CBVN preservation has been expected to provide patients with more favorable nutritional status and decreasing risk of gallstones by improving gastrointestinal function controlled by the autonomic nerves [13], although it has been feared that the CBVN preservation disturbs the lymph node dissection along the left gastric artery. Recently, a randomized controlled trial has revealed that the CBVN preservation in distal gastrectomy significantly diminishes diarrhea and appetite loss at 12 months post surgery and does not affect the long-term outcome compared with that associated with no preservation of the CBVN [14].

Based on these data concerning PPG and the CBVN preservation, a clinical question arose whether the CBVN preservation also provided patients with more clinical benefit in PPG. Several studies have attempted to demonstrate that CBVN preservation contributes to improvement of dumping syndrome and nutritional status or is oncologically safe in PPG [15-18]. However, all those studies were retrospective studies with small sample sizes, and they have not clearly demonstrated whether the CBVN preservation truly provides patients with benefits in PPG.

In the present study, to clarify the clinical, short-term, and long-term benefits of the CBVN preservation in PPG, the surgical and oncological outcomes and nutritional status of patients undergoing LPPG with preservation of the CBVN compared with those findings for patients without the CBVN preservation were retrospectively evaluated. To address many selection biases existing in the retrospective comparison between the groups with and without the CBVN preservation, a case-matching analysis was performed between the two groups on the basis of the estimated propensity scores of each patient.

\section{Patients and methods}

\section{Patients}

From January 2005 to December 2010, 391 consecutive patients with histologically verified gastric adenocarcinoma underwent LPPG with curative intent in the Department of Gastroenterological Surgery, Cancer Institute Hospital, Tokyo, Japan. All tumors were diagnosed as mucosal or submucosal adenocarcinomas without lymph node metastasis (cT1, cN0), on the basis of preoperative examinations, including upper gastrointestinal endoscopy, barium radiography, computed tomography, and sometimes endoscopic or abdominal ultrasonography. In the authors' institution, the LPPG procedure has been indicated for $\mathrm{cT} 1$, cN0 gastric cancer located in the middle third of the stomach more than $5 \mathrm{~cm}$ proximal to the pyloric ring and with a maximum diameter less than $5 \mathrm{~cm}$. Patients older than 75 years of age were excluded from this study because we considered that pyloric function was frequently insufficient in elderly patients. Of the 391 patients, 304 underwent LPPG with the CBVN preservation, and the remaining 87 underwent LPPG without the CBVN preservation. During this period, whether the CBVN was preserved depended on each surgeon's preference. Written informed consent was obtained from all patients before surgery.

\section{Surgical procedure}

Pneumoperitoneum was created by the injection of carbon dioxide at $10-12 \mathrm{mmHg}$, and a laparoscope was inserted through the umbilical port. Under the view of the laparoscopic image, four ports (each 5-12 mm) were inserted into the left upper, left flank, right upper, and right flank quadrants. The LPPG technique consisted of the following procedures, as previously described $[6,17]$, with $\mathrm{D} 1+$ lymphadenectomy performed according to the 2 nd edition of the Japanese Classification of Gastric Cancer (JCGC) [19]. A summary of our LPPG procedures is as follows: (1) the greater omentum was dissected; (2) the right gastroepiploic vein and artery were divided while preserving the infrapyloric vessels to maintain blood supply to the remaining pyloric cuff; (3) the suprapyloric lymph nodes were picked up if required, and the right gastric artery and the pyloric branch of the vagal nerve were preserved; (4) the lesser omentum was dissected, and the hepatic branch of the vagal nerve was preserved; (5) the trunk of the left gastroepiploic artery and vein were divided; (6) the greater curvature was rolled up toward the abdominal wall, and the suprapancreatic lymph nodes along the common hepatic artery and splenic artery were dissected; (7) when the CBVN was not preserved, the root of the left gastric artery was divided; (8) when the CBVN was preserved, the CBVN was made visible from the left side of the gastropancreatic ligament, the left gastric artery was divided at the distal site where the CBVN met, the CBVN was carefully skeletonized using an ultrasonically activated device so as not to cause thermal injury [20], and the posterior gastric branch of the vagal nerve was cut [17]; (9) the cardiac lymph nodes and the lymph nodes along the lesser curvature of the stomach were dissected; and (10) the stomach was transected $3-5 \mathrm{~cm}$ proximal to the pylorus and at the proximal site of the tumor, depending on the negative-confirmation biopsy. After the transection of the stomach, a gastro-gastro anastomosis was made by hand-suturing or using a linear stapler.

\section{Data collection and analysis}

To evaluate the surgical and oncological safety of the CBVN preservation procedure, surgical and early postoperative outcomes such as operation time, blood loss, 
postoperative hospital stay and postoperative complications, pathological findings, and the postoperative recurrence rate were compared between the patients whose CBVN was preserved in LPPG (CBP group) and those whose CBNV was cut (non-CBP group). Postoperative complications were classified according to the Clavien-Dindo classification of surgical complications [21], and complications of grade II and above were reviewed. For the evaluation of the clinical benefits of the procedure, postoperative nutritional status and functions were analyzed. In evaluating nutritional status, changes in body weight and the serum level of total protein, albumin, and level of hemoglobin were compared between the two groups. Endoscopic examinations were performed 1 year after surgery to evaluate the postoperative function of the remnant stomach such as reflux esophagitis, gastritis, bile reflux, and the presence of residual food according to the RGB (residue, gastritis, bile) classification, and those of grade 1 or higher were recorded [22]. Gallstone formation after surgery was routinely examined using abdominal ultrasonography or computed tomography once or twice per year. All the data regarding patient characteristics, operation details, pathological reports, hospital course, and morbidity were retrospectively collected. Pathological stages were determined according to the third edition of JCGC [23].

\section{Propensity score estimation and case matching}

To address the selection bias that is inherent in retrospective observational studies, a case-matching analysis was performed between the $\mathrm{CBP}$ and non-CBP groups on the basis of the estimated propensity score of each patient. The score was estimated using a logistic regression model and optimal matching (ratio 1:2) with a caliper of width 0.2 standard deviations of the logit of the estimated propensity score. These following factors were inserted as covariates: sex, body mass index (BMI), year of operation, clinical $\mathrm{T}$ stage, tumor size, and surgeon. The score estimation and case matching were derived by one specialized clinical researcher who was blind to the outcomes.

\section{Statistical analysis}

Statistical differences between two groups were calculated with the Student's $t$ test, Mann-Whitney $U$ test, or chisquared test and Fisher's exact test as appropriate. Statistical significance was defined as $P<0.05$. The statistical analysis was performed with the JMP (version 11.0) software program (SAS Institute, Cary, NC, USA).

\section{Results}

\section{Patient characteristics after propensity score matching}

Data for 116 patients in the CBP group and 58 patients in the non-CBP group were selected using the propensity scorematching method. The clinical characteristics of the patients in the two groups are presented in Table 1. The two groups were comparable with regard to the variables (age, gender, BMI, clinical T stage, operator) considered for propensity score matching. Additionally, the sizes and pathological data of the tumors were similar in both groups. Although all preoperative diagnoses were $\mathrm{T} 1$ stage, histological analysis of the depth of cancer invasion demonstrated that 11 (9.4\%) in the CBP group and $3(5.1 \%)$ in the non-CBP group had a pT2 or deeper than pT2 stage tumor. Fourteen patients $(12.1 \%)$ in the CBP group and 5 patients $(8.4 \%)$ in the nonCBP group had lymph node metastasis. The incidence of metastasis in each lymph node station dissected in the two groups is presented in Table 2 .

\section{Short-term outcomes}

Table 3 summarizes the short-term outcomes of the CBP and non-CBP groups. The most frequent complication in both

Table 1 Baseline patient characteristics

\begin{tabular}{llll}
\hline Characteristic & CBP $(n=116)$ & Non-CBP $(n=58)$ & $P$ \\
\hline Age & $59[30-80]$ & $58[38-84]$ & 0.519 \\
Sex & & & \\
$\quad$ Male/female & $70 / 46$ & $43 / 15$ & 0.103 \\
BMI, kg/m ${ }^{2}$ & $23.6[19.5-30.9]$ & $24.2[16.8-30.1]$ & 0.098 \\
cT stage & & & \\
$\quad$ T1a/T1b & $56 / 60$ & $29 / 29$ & 0.957 \\
Operator & & & \\
A & 27 & 10 & 0.144 \\
B & 21 & 18 & \\
$\quad$ Others & 68 & 30 & 0.425 \\
Tumor size, mm & $25[7-90]$ & $25[6-90]$ & \\
Histological type & & & 0.176 \\
Differentiated & 36 & 24 & 0.373 \\
Undifferentiated & 80 & 34 & \\
pT stage & & & \\
T1a/T1b/T2/T3/T4a & $64 / 41 / 4 / 5 / 2$ & $27 / 28 / 0 / 2 / 1$ & \\
pN stage & & $53 / 3 / 1 / 1$ & \\
N0/N1/N2/N3 & $102 / 11 / 3 / 0$ & & \\
ESD before surgery & & $9 / 109$ & \\
Yes/no & & & \\
\hline
\end{tabular}

$C P B$ celiac branch preservation, $B M I$ body mass index, ESD endoscopic mucosal dissection

Data are presented as median [range] 


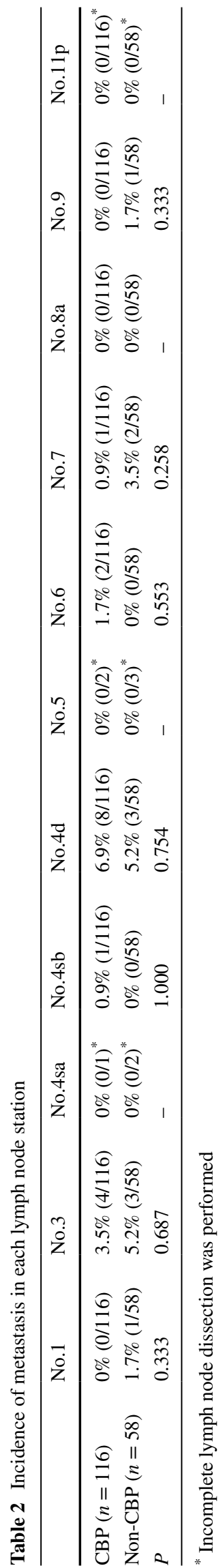

the CBP (7.8\%) and non-CBP groups (5.2\%) was delayed gastric emptying. There were no significant differences in the incidence of postoperative complications between the two groups. In both groups, the operative mortality rate was $0 \%$. There were no significant differences between two groups in operation time ( 237 vs. $235.5 \mathrm{~min}, P=0.670$ ) and intraoperative blood loss (30 vs. $40 \mathrm{~g}, P=0.137$ ). D1+ lymph node dissection was performed for all patients, and the median numbers of retrieved lymph nodes were similar between the groups. No conversion to open surgery occurred in either group. For the postoperative course, time to first flatus, time to the start of oral intake, and time of postoperative stay were similar between the two groups.

\section{Long-term outcomes}

\section{Oncological outcomes}

To assess survival and recurrence rates, the median followup periods for patients in the CBP and non-CBP groups were 60 months in both groups (Table 4). The 5-year overall survival rates were $98.2 \%$ in the CBP group and $96.5 \%$ in the non-CBP group. One patient with pT4aN0 gastric cancer in the CBP group developed peritoneal recurrence, and one patient with pT1bN3a in the non-CBP group developed bone recurrence.

\section{Remnant stomach function, nutrition, and gallstone formation}

Table 5 shows an endoscopic evaluation 12 months after surgery. Five patients in the CBP group and five patients in the non-CBP group did not undergo gastrointestinal endoscopy 1 year after surgery. Almost half the patients in both groups had some food residue in the gastric remnant and residual gastritis. The incidences of esophageal and bile reflux were similar in the two groups. The parameters of nutritional status (body weight change, level of hemoglobin, total protein, and albumin) were not significantly different between the two groups during the 5 years of the follow-up period (Fig. 1). Gallstones developed in eight (7.8\%) patents (seven men and one woman) in the CBP group and five (10.6\%) men in the non-CBP group. Although the patients in the non-CBP group experienced postoperative gallstone formation more frequently than the patients in the CBP group, there was no significant difference $(P=0.758)$.

\section{Discussion}

PPG was first performed as a treatment for gastric ulcers in 1967 [24]. Recently, LPPG has become one of the surgical modalities used for early gastric cancer to reduce invasion 
Table 3 Surgical and postoperative outcomes

\begin{tabular}{llll}
\hline Variable & CBP $(n=116)$ & Non-CBP $(n=58)$ & $P$ \\
\hline Operation time, min & $237[140-373]$ & $235.5[154-407]$ & 0.670 \\
Blood loss, g & $30[3-440]$ & $40[0-350]$ & 0.137 \\
Total number of resected lymph nodes & $34[17-70]$ & $33.5[14-70]$ & 0.457 \\
Postoperative complication (\%) & & & \\
Bleeding & $1(0.86)$ & $0(0)$ & 1.000 \\
Pancreatic fistula & $5(5.8)$ & $5(8.6)$ & 0.304 \\
Anastomotic leakage & $1(0.86)$ & $0(0)$ & 1.000 \\
Delayed gastric emptying & $9(7.8)$ & $3(5.2)$ & 1.000 \\
Bowel obstruction & $0(0)$ & $1(1.7)$ & 0.333 \\
Time until start of flatus, days & $2[0-4]$ & $2[1-4]$ & 0.686 \\
Time until start of oral intake, days & $2[2-4]$ & $2[2-4]$ & 0.204 \\
Postoperative stay, days & $11[8-51]$ & $12[7-55]$ & 0.312 \\
\hline
\end{tabular}

Data are presented as median [range]

$C B P$ celiac branch preserving group

\begin{tabular}{llll}
\hline Variable & CBP $(n=116)$ & Non-CBP $(n=58)$ & $P$ \\
\hline Median follow-up period, months [range] & $60[2-112]$ & $60[6-108]$ & 0.694 \\
Recurrence (\%) & $1(0.9)$ & $1(1.7)$ & 1.000 \\
5-year overall survival rate, \% & 98.2 & 96.5 & 0.468 \\
5-year recurrence-free survival rate, $\%$ & 99.1 & 97.1 & 0.844 \\
\hline
\end{tabular}

Table 4 Recurrence and survival the basis of the estimated propensity score of each patient. The surgical and short-term outcomes, including operation time, blood loss, surgical morbidity, and mortality, were similar in the two groups. For long-term outcomes, such as oncological safety, remnant stomach function, nutritional status, and gallstone formation, there were no significant differences between the two groups. Therefore, no apparent advantage of the CBVN preservation in LPPG compared with nonpreservation was found. This is the first report of a comparative evaluation of not only short-term outcomes but also long-term outcomes of the CBVN preservation by a case-matching analysis based on the data of nearly 400 patients undergoing LPPG.

The surgical technique of the CBVN preservation in LPPG was found to be safe and feasible in terms of intraoperative and early postoperative outcomes. The preservation of the CBVN might increase the difficulty of the LPPG procedure, because the lymph nodes in the gastropancreatic ligament must be dissected and the CBVN must be identified and preserved along with the left gastric artery connected to the CBVN. However, the surgical outcomes, such as length of operation, amount of blood loss, postoperative complications, and total number of retrieved lymph nodes in the present study were similar between the CBP and non-CBP groups. The additional procedures for preserving the CBVN did not affect technical difficulty and harvesting of lymph nodes in LPPG. One of the possible reasons why the CBVN 
Fig. 1 Preoperative and postoperative body weight changes and levels of hemoglobin, total protein, and albumin in the CBP and non-CBP groups. There were no significant differences in nutritional status between the two groups during the 5-year postoperative period. Filled circles $\mathrm{CBP}$ group, open circles non-CBP group. Error bar represents standard deviation
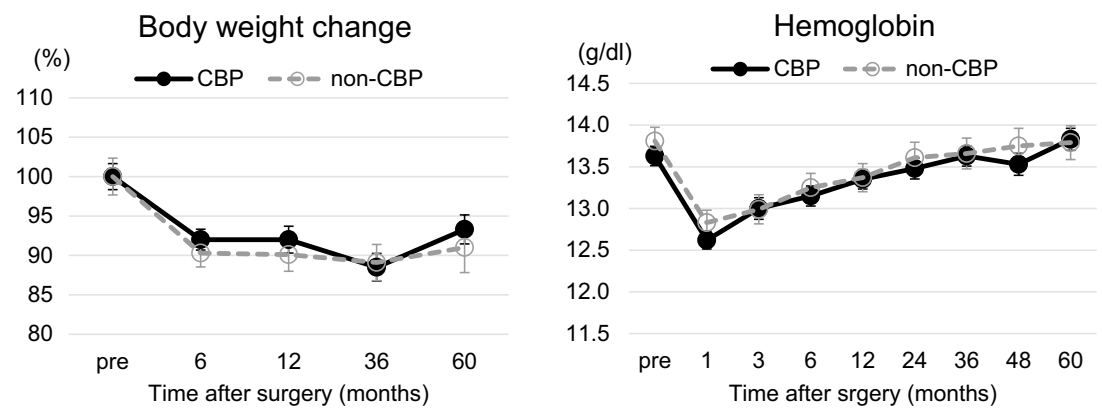

Total Protein

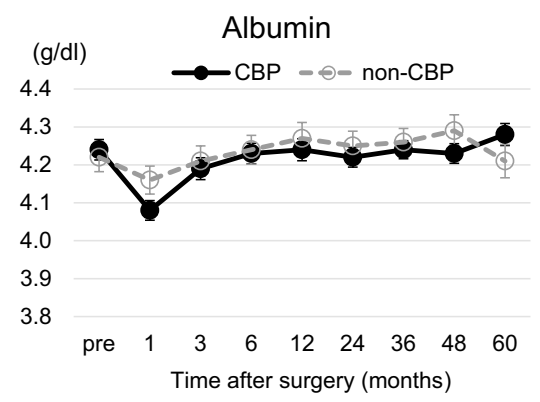

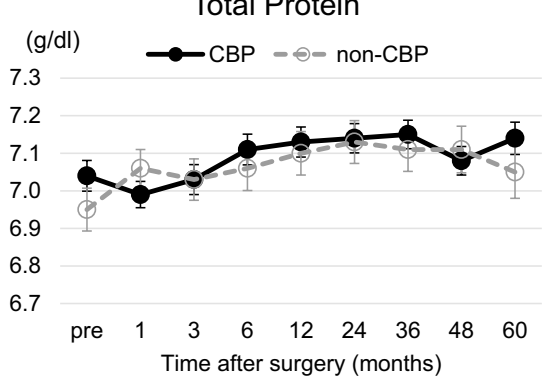

preservation did not affect the operative results is that all the patients in this study underwent LPPG performed by experts in Japan who perform more than 100 laparoscopic gastrectomies per year.

Moreover, the present study showed that the CBVN preservation in LPPG is oncologically safe. Although we had 14 patients with pT2 or deeper, and 21 with lymph node metastasis, only one peritoneal relapse $(0.9 \%)$ occurred in the CBP group; this result is quite reasonable compared with previous reports $[10,25]$. The relapse might be not associated with the CBVN preservation in LPPG, because the patient had advanced gastric cancer with serosal invasion and no lymph node metastasis. Regarding oncological safety, the dissection technique of the lymph nodes along the left gastric artery in the CBVN preservation might be considered inadequate because the fatty tissue containing these lymph nodes is split for preservation of the nerve. However, the results in this study demonstrated that there were no differences in survival and the total number of retrieved lymph nodes between the two groups.

We expected that the CBVN preservation in LPPG relieved some symptoms of postgastrectomy syndrome, such as dumping syndrome and bile reflux, and also relieved the disadvantage of PPG, delayed gastric emptying, which induced more favorable nutritional status. Postgastrectomy syndrome might be related to not only gastrectomy but also to resection of the CBVN to some extent because the CBVN innervates the gastrointestinal tract and is associated with pancreatic insulin release. Moreover, PPG is well known to reduce symptoms of postgastrectomy syndrome by preventing food from dumping, whereas the incidence of delayed gastric emptying is more frequent than that after distal gastrectomy [9, 15, 28-35]. However, in the present study, none of the expected benefits associated with postoperative nutritional status were found over the course of 5 years. Furthermore, delayed gastric emptying, endoscopic findings a year after LPPG, such as the presence of residual food, remnant gastritis, and the incidence of gallstone formation, were not different between the groups, although previous studies have reported that the preservation of the vagal nerve maintains gastrointestinal motility and prevents postoperative gallstones [11, 12]. In terms of patient symptoms, Kim et al. have reported that preserving the vagal nerve attenuates the occurrence of diarrhea and appetite loss after distal gastrectomy [14]. Because the present study was retrospective and observational, the postoperative symptoms of our patients could not be precisely evaluated. However, nutritional status is presumably one of the surrogate markers that represent the degree of diarrhea and appetite, because diarrhea and appetite loss easily induce body weight loss and hypoproteinemia [36]. There is no doubt that the occurrence of diarrhea and appetite loss was not associated with the CBVN preservation in LPPG because all nutritional parameters evaluated in this study were identical in both groups during the long observation period.

Why were there no advantages in nutritional status, remnant stomach function, and gallstone formation, even though these benefits have been observed in distal gastrectomy [11, 12]? It is presumable that the major reason is that LPPG has several advantages compared to conventional distal gastrectomy in terms of postoperative function, such as the prevention of dumping syndrome, bile juice reflux, gallbladder contraction, and nutritional status, as the result of pylorus preservation accompanying the hepatic branch of the vagal 
nerve $[9,15,28-35]$. In LPPG, the CBVN preservation did not produce benefits because LPPG itself has many advantages, as already mentioned, as a function-preserving surgery [35]. The procedure of the CBVN preservation in LPPG did not confer additional advantages over those conferred by preservation of the pylorus and the hepatic branch of the vagus.

This study has several limitations. First, this study was retrospectively conducted in a single institution, although certain limitations such as uneven demographic data regarding patient characteristics were resolved by using propensity score matching. Second, we were unable to evaluate postoperative symptoms, including diarrhea or gastric fullness, which are decreased by the CBVN preservation in distal gastrectomy. This limitation is critical in this study, because the nutritional status might be a surrogate marker of such important symptoms. Third, the lack of statistical power may have resulted in the lack of observed differences between the groups in this study. A larger size study or a longer follow-up might reveal why LPPG with the CBVN preservation was superior to that without preservation. According to these limitations, our results in the present study are not definitive.

In conclusion, the procedure of preserving the $\mathrm{CBVN}$ in LPPG by a skillful surgeon is safe and feasible on the basis of short- and long-term outcomes. However, the preservation procedure might not produce any clinical benefits in terms of remnant gastric function, gallstone formation, and nutritional indicators compared with cases with no preservation. It is presumable that the CBVN preservation does not provide patients with additional clinical benefits over those that PPG provides. Considering the results and discussion in this study, further investigation into the clinical significance of the CBVN preservation in LPPG might not be required.

\section{Compliance with ethical standards}

Conflict of interest The authors declare no conflict of interest.

Human rights statement and informed consent All procedures performed in studies involving human participants were in accordance with the ethical standards of the institutional and/or national research committee and with the 1964 Helsinki Declaration and its later amendments or comparable ethical standards. Informed consent was obtained from all individual participants included in the study.

\section{References}

1. Jemal A, Bray F, Center MM, Ferlay J, Ward E, Forman D. Global cancer statistics. CA Cancer J Clin. 2011;61:69-90.

2. Hamashima C, Okamoto M, Shabana M, Osaki Y, Kishimoto T. Sensitivity of endoscopic screening for gastric cancer by the incidence method. Int J Cancer. 2013;133:653-9.

3. Maehara Y, Kakeji Y, Oda S, Takahashi I, Akazawa K, Sugimachi K. Time trends of surgical treatment and the prognosis for Japanese patients with gastric cancer. Br J Cancer. 2000;83:986-91.

4. Kodera Y, Yamamura Y, Kanemitsu Y, Shimizu Y, Hirai T, Yasui $\mathrm{K}$, et al. Lymph node metastasis in cancer of the middle-third stomach: criteria for treatment with a pylorus-preserving gastrectomy. Surg Today. 2001;31:196-203.

5. Nashimoto A, Akazawa K, Isobe Y, Miyashiro I, Katai H, Kodera Y, et al. Gastric cancer treated in 2002 in Japan: 2009 annual report of the JGCA nationwide registry. Gastric Cancer. 2013;16:1-27.

6. Hiki N, Shimoyama S, Yamaguchi H, Kubota K, Kaminishi M. Laparoscopy-assisted pylorus-preserving gastrectomy with quality controlled lymph node dissection in gastric cancer operation. J Am Coll Surg. 2006;203:162-9.

7. Kim BH, Hong SW, Kim JW, Choi SH, Yoon SO. Oncologic safety of pylorus-preserving gastrectomy in the aspect of micrometastasis in lymph nodes at stations 5 and 6. Ann Surg Oncol. 2014;21:533-8.

8. Kumagai K, Hiki N, Nunobe S, Sekikawa S, Chiba T, Kiyokawa $\mathrm{T}$, et al. Totally laparoscopic pylorus-preserving gastrectomy for early gastric cancer in the middle stomach: technical report and surgical outcomes. Gastric Cancer. 2015;18:183-7.

9. Zhang D, Shimoyama S, Kaminishi M. Feasibility of pyloruspreserving gastrectomy with a wider scope of lymphadenectomy. Arch Surg. 1998;133:993-7.

10. Ikeguchi M, Hatada T, Yamamoto M, Miyake T, Matsunaga T, Fukuda K, et al. Evaluation of a pylorus-preserving gastrectomy for patients preoperatively diagnosed with early gastric cancer located in the middle third of the stomach. Surg Today. 2010;40:228-33.

11. Kojima K, Yamada H, Inokuchi M, Kawano T, Sugihara K. Functional evaluation after vagus-nerve-sparing laparoscopically assisted distal gastrectomy. Surg Endosc. 2008;22:2003-8.

12. Yamada H, Kojima K, Inokuchi M, Kawano T, Sugihara K. Efficacy of celiac branch preservation in Roux-en-Y reconstruction after laparoscopy-assisted distal gastrectomy. Surgery (St. Louis). 2011;149:22-8.

13. Inokuchi M, Sugita H, Otsuki S, Sato Y, Nakagawa M, Kojima K. Long-term effectiveness of preserved celiac branch of vagal nerve after Roux-en-Y reconstruction in laparoscopy-assisted distal gastrectomy. Dig Surg. 2014;31:341-6.

14. Kim SM, Cho J, Kang D, Oh SJ, Kim AR, Sohn TS, et al. A randomized controlled trial of vagus nerve-preserving distal gastrectomy versus conventional distal gastrectomy for postoperative quality of life in early stage gastric cancer patients. Ann Surg. 2016;263:1079-84

15. Isozaki H, Okajima K, Momura E, Ichinona T, Fujii K, Izumi N, et al. Postoperative evaluation of pylorus-preserving gastrectomy for early gastric cancer. Br J Surg. 1996;83:266-9.

16. Shinohara H, Sonoda T, Niki M, Nomura E, Nishiguchi K, Tanigawa N. Laparoscopically-assisted pylorus-preserving gastrectomy with preservation of the vagus nerve. Eur J Surg. 2002;168:55-8.

17. Nunobe S, Hiki N, Fukunaga T, Tokunaga M, Ohyama S, Seto $\mathrm{Y}$, et al. Laparoscopy-assisted pylorus-preserving gastrectomy: preservation of vagus nerve and infrapyloric blood flow induces less stasis. World J Surg. 2007;31:2335-40.

18. Tokunaga M, Hiki N, Fukunaga T, Ohyama S, Nunobe S, Yamada $\mathrm{K}$, et al. Is preservation of the celiac branch of the vagal nerve effective in preventing stasis following pylorus-preserving gastrectomy? Hepatogastroenterology. 2011;58:1046-50.

19. Japanese Gastric Cancer Association. Japanese classification of gastric carcinoma, 2nd English edition. Gastric Cancer. $1998 ; 1: 10-24$ 
20. Irino T, Hiki N, Ohashi M, Nunobe S, Sano T, Yamaguchi T. The Hit and Away technique: optimal usage of the ultrasonic scalpel in laparoscopic gastrectomy. Surg Endosc. 2016;30:245-50.

21. Dindo D, Demartines N, Clavien PA. Classification of surgical complications: a new proposal with evaluation in a cohort of 6336 patients and results of a survey. Ann Surg. 2004;240:205-13.

22. Kubo M, Sasako M, Gotoda T, Ono H, Fujishiro M, Saito D, et al. Endoscopic evaluation of the remnant stomach after gastrectomy: proposal for a new classification. Gastric Cancer. 2002;5:83-9.

23. Japanese Gastric Cancer Association. Japanese classification of gastric carcinoma, 3rd English edition. Gastric Cancer. 2011;14:101-12.

24. Maki T, Shiratori T, Hatafuku T, Sugawara K. Pylorus-preserving gastrectomy as an improved operation for gastric ulcer. Surgery (St. Louis). 1967;61:838-45.

25. Morita S, Katai H, Saka M, Fukagawa T, Sano T, Sasako M. Outcome of pylorus-preserving gastrectomy for early gastric cancer. Br J Surg. 2008;95:1131-5.

26. Matsuki A, Nashimoto A, Yabusaki H, Nakagawa S. Long-term clinical outcome and survival after pylorus-preserving gastrectomy. Hepatogastroenterology. 2012;59:2012-5.

27. Hiki N, Nunobe S, Kubota T, Jiang X. Function-preserving gastrectomy for early gastric cancer. Ann Surg Oncol. 2013;20:2683-92.

28. Kodama M, Koyama K. Indications for pylorus preserving gastrectomy for early gastric cancer located in the middle third of the stomach. World J Surg. 1991;15:628-33 (discussion 33-4).

29. Imada T, Rino Y, Takahashi M, Suzuki M, Tanaka J, Shiozawa $\mathrm{M}$, et al. Postoperative functional evaluation of pylorus-preserving gastrectomy for early gastric cancer compared with conventional distal gastrectomy. Surgery (St. Louis). 1998;123:165-70.

30. Nishikawa K, Kawahara H, Yumiba T, Nishida T, Inoue Y, Ito T, et al. Functional characteristics of the pylorus in patients undergoing pylorus-preserving gastrectomy for early gastric cancer. Surgery (St. Louis). 2002;131:613-24.

31. Shimoyama S, Mafune K, Kaminishi M. Indications for a pyloruspreserving gastrectomy for gastric cancer with proper muscle invasion. Arch Surg. 2003;138:1235-9.

32. Yamaguchi T, Ichikawa D, Kurioka H, Ikoma H, Koike H, Otsuji $\mathrm{E}$, et al. Postoperative clinical evaluation following pylorus-preserving gastrectomy. Hepatogastroenterology. 2004;51:883-6.

33. Hotta T, Taniguchi K, Kobayashi Y, Johata K, Sahara M, Naka T, et al. Postoperative evaluation of pylorus-preserving procedures compared with conventional distal gastrectomy for early gastric cancer. Surg Today. 2001;31:774-9.

34. Nunobe S, Sasako M, Saka M, Fukagawa T, Katai H, Sano T. Symptom evaluation of long-term postoperative outcomes after pylorus-preserving gastrectomy for early gastric cancer. Gastric Cancer. 2007;10:167-72.

35. Suh YS, Han DS, Kong SH, Kwon S, Shin CI, Kim WH, et al. Laparoscopy-assisted pylorus-preserving gastrectomy is better than laparoscopy-assisted distal gastrectomy for middle-third early gastric cancer. Ann Surg. 2014;259:485-93.

36. Adachi S, Takiguchi S, Okada K, Yamamoto K, Yamasaki M, Miyata $\mathrm{H}$, et al. Effects of ghrelin administration after total gastrectomy: a prospective, randomized, placebo-controlled phase II study. Gastroenterology. 2010;138:1312-20. 